\title{
LARGE DEVIATION PROBABILITIES FOR THE NUMBER OF VERTICES OF RANDOM POLYTOPES IN THE BALL
}

\author{
PIERRE CALKA, ${ }^{*}$ Université René Descartes Paris 5 \\ TOMASZ SCHREIBER, ${ }^{* *}$ Nicolaus Copernicus University, Toruń
}

\begin{abstract}
In this paper we establish large deviation results on the number of extreme points of a homogeneous Poisson point process in the unit ball of $\mathbb{R}^{d}$. In particular, we deduce an almost-sure law of large numbers in any dimension. As an auxiliary result we prove strong localization of the extreme points in an annulus near the boundary of the ball.

Keywords: Convex hull; covering of the sphere; large deviation; measure concentration; random polytope
\end{abstract}

2000 Mathematics Subject Classification: Primary 60D05

Secondary 60F 10

\section{Introduction and main results}

Let us denote by $U_{1}, \ldots, U_{n}, n \in \mathbb{N}^{*}=\mathbb{N} \backslash\{0\}, n$ independent, uniformly distributed variables in the unit ball, $\mathbb{B}^{d}$, of the Euclidean space $\mathbb{R}^{d}, d \geq 2$, and let $X_{t}, t>0$, be a homogeneous Poisson point process in $\mathbb{B}^{d}$ of intensity measure $\left(t / \omega_{d}\right) \mathbf{1}_{\mathbb{B}^{d}}(x) \mathrm{d} x$, where $\omega_{d}$ and $\mathbf{1}_{\mathbb{B}^{d}}(\cdot)$ are respectively the volume and the indicator function of the unit ball. We consider both the respective convex hulls, $C_{n}$ and $\tilde{C}_{t}$, of $\left\{U_{1}, \ldots, U_{n}\right\}$ and $X_{t}$, and $N_{n}$ and $\tilde{N}_{t}$, the numbers of vertices in each convex hull. The asymptotic behaviour of $N_{n}$ as $n \rightarrow \infty$ has been widely investigated in the literature. For $d=2$, Rényi and Sulanke [11] in 1963 obtained the convergence of means of $N_{n}$. Their work has been followed by Efron [6], Buchta and Müller [3], Groeneboom [7], who obtained a central limit theorem, and Massé [9], who proved a law of large numbers in probability. More recently, by precisely estimating the variance of $N_{n}$ for all dimensions $d \geq 2$, Reitzner [10] deduced an almost-sure convergence for the number of vertices of random polyhedra in any convex set of $\mathbb{R}^{d}, d \geq 4$, with a twice-continuously differentiable boundary and positive Gaussian curvature. For all $d \geq 2$, the asymptotic behaviour of $\mathrm{E}\left(N_{n}\right)$ is known to be

$$
\mathrm{E}\left(N_{n}\right) \sim c_{d} n^{(d-1) /(d+1)},
$$

where the dimension-dependent constant $c_{d}$ is known explicitly; see [14] (for $d=3$ ), [1], and [13], as well as Equation (7) of [10] and the references therein. Note that $\alpha_{s} \sim \beta_{s}$ means $\lim _{s \rightarrow \infty} \alpha_{s} / \beta_{s}=1$. Throughout the paper we make use of ' $O, \Omega$, $\Theta$ ' notation: recall that $O(X)$ stands for quantities bounded above by a constant multiple of $X, \Omega(X)$ for quantities bounded below by a constant multiple of $X$, and $\Theta(X)=O(X) \cap \Omega(X)$.

Received 4 January 2005; revision received 14 November 2005.

* Postal address: Université René Descartes Paris 5, MAP5, UFR Math-Info, 45 rue des Saints-Pères, 75270 Paris Cedex 06, France. Email address: pierre.calka@math-info.univ-paris5.fr

** Postal address: Faculty of Mathematics and Computer Science, Nicolaus Copernicus University, ul. Chopina 12/18, 87-100 Toruń, Poland. Email address: tomeks@mat.uni.torun.pl 
Note that (1) implies the same type of asymptotics for $\tilde{N}_{t}$ as $t \rightarrow \infty$, i.e.

$$
\mathrm{E}\left(\tilde{N}_{t}\right) \sim c_{d} t^{(d-1) /(d+1)} .
$$

Indeed, because a homogeneous Poisson process in $\mathbb{B}^{d}$ of intensity $t$ coincides in distribution with $\left\{U_{1}, \ldots, U_{S}\right\}$, where $S$ is a Poisson variable with mean $t$, we obtain

$$
\begin{aligned}
\left|\mathrm{E}\left(\tilde{N}_{t}\right)-\sum_{|k-t| \leq t^{2 / 3}} \mathrm{E}\left(N_{k}\right) \mathrm{P}\{S=k\}\right| & \leq \sum_{|k-t| \leq t^{2 / 3}} k \mathrm{P}\{S=k\} \\
& =t \mathrm{P}\left\{S \notin\left[t-1-t^{2 / 3}, t-1+t^{2 / 3}\right]\right\} .
\end{aligned}
$$

It remains to use standard moderate deviation results on the Poisson distribution to prove that the right-hand side of this expression goes to 0 as $t \rightarrow \infty$, and that the summation on the left-hand side tends to $c_{d} t^{(d-1) /(d+1)}$.

The purpose of this paper is to establish the following large deviation results for $N_{n}$ and, respectively, $\tilde{N}_{t}$.

Theorem 1. For each $\varepsilon>0$,

$$
\liminf _{n \rightarrow \infty} \frac{1}{\log n} \log \left(-\log \mathrm{P}\left\{\left|\frac{N_{n}}{\mathrm{E}\left(N_{n}\right)}-1\right|>\varepsilon\right\}\right) \geq \frac{d-1}{3 d+5} .
$$

Theorem 2. For each $\varepsilon>0$,

$$
\liminf _{t \rightarrow \infty} \frac{1}{\log t} \log \left(-\log \mathrm{P}\left\{\left|\frac{\tilde{N}_{t}}{\mathrm{E}\left(\tilde{N}_{t}\right)}-1\right|>\varepsilon\right\}\right) \geq \frac{d-1}{3 d+5}
$$

Note that these results are of the same type as the concentration results for volumes of unions of random closed sets obtained in [12]. The authors believe that the concentration rate, $(d-1) /(3 d+5)$, on the right-hand sides of (2) and (3) is not optimal, and conjecture that the optimal value should be $(d-1) /(d+1)$, coinciding with the exponent determining the asymptotics of the expected number of vertices. However, we were not able to verify this conjecture using our current methods.

In particular, we deduce the following almost-sure laws of large numbers for $N_{n}$ and, respectively, $\tilde{N}_{t}$, in any dimension $d \geq 2$.

Corollary 1. We have

$$
\lim _{n \rightarrow \infty} \frac{N_{n}}{\mathrm{E}\left(N_{n}\right)}=1 \quad \text { almost surely }
$$

and

$$
\lim _{t \rightarrow \infty} \frac{\tilde{N}_{t}}{\mathrm{E}\left(\tilde{N}_{t}\right)}=1 \quad \text { almost surely. }
$$

Corollary 1 is a direct consequence of Theorems 1 and 2 and the Borel-Cantelli lemma.

Our technique of proof strongly relies on the localization of extreme points in a small annulus near the boundary of the unit ball, which allows us to use a standard concentration-of-measure result due to Ledoux [8, pp. Corollary 1.17]. The following proposition shows that, with an overwhelming probability going exponentially quickly to 1 , the vertices of the convex hull of the points inside the ball are located in an annulus, centred at the origin, of thickness of order 
$n^{-2 /(d+1)}$ (for $C_{n}$ ) or $t^{-2 /(d+1)}$ (for $C_{t}$ ). The method of proof of this result is based on a classically known technique of cap coverings that was first developed and exploited in [2].

In what follows, $B(r), r>0$, denotes the ball of radius $r$ centred at the origin, and we generically use $c$ to denote some positive constant.

Proposition 1. (i) There exist constants, $c>0$ and $K>0$, such that, for every $\alpha, 0<\alpha<$ $2 /(d+1)$, we have

$$
\mathrm{P}\left\{B\left(1-K n^{-\alpha}\right) \nsubseteq C_{n}\right\}=O\left(\exp \left(-c n^{1-\alpha(d+1) / 2}\right)\right) .
$$

(ii) For the same $c$ and $K$, we have

$$
\mathrm{P}\left\{B\left(1-K t^{-\alpha}\right) \nsubseteq \tilde{C}_{t}\right\}=O\left(\exp \left(-c t^{1-\alpha(d+1) / 2}\right)\right) .
$$

Our main motivation is the extension to higher dimensions $(d \geq 2)$ of the authors' previous results on the number of sides [5] and the radius of the circumscribed ball [4] of the typical Poisson-Voronoi cell in the Euclidean plane. Indeed, in those papers we established a connection between the sides of the typical cell and the extreme points of an inhomogeneous Poisson point process in the unit ball, via an action of the classical inversion. In dimension $d \geq 3$, the same argument provides a relation between the number of hyperfaces or the radius of the circumscribed ball of the typical cell and the number of vertices or, respectively, the inradius of the convex hull of the Poisson process inside the ball. We will deduce from Theorem 2, Corollary 1, and Proposition 1 some new results on the geometry of the typical Poisson-Voronoi cell that will be developed in future work.

The paper is structured as follows. We first prove an auxiliary proposition stating the localization of the extreme points near the boundary of the ball. By using concentrationof-measure arguments due to Ledoux [8], we then prove the main large deviation result for the number $N_{n}$ (see Theorem 1). We deduce Theorem 2 from Theorem 1 and a large deviation property of the Poisson distribution. Finally, we make some concluding remarks about the possible extensions of these results.

\section{Proof of Proposition 1.}

Proof of part (i). For a fixed $u_{0} \in \mathbb{S}^{d-1}\left(\mathbb{S}^{d-1}\right.$ being the unit sphere of $\mathbb{R}^{d}$ ), let $S_{n}=$ $\sup _{1 \leq i \leq n}\left(U_{i} \cdot u_{0}\right)$, where ' $'$ ' denotes the usual scalar product in $\mathbb{R}^{d}$. There exists a $c>0$ such that, for every $\alpha \in(0,2 /(d+1))$, we have

$$
\mathrm{P}\left\{S_{n} \leq 1-n^{-\alpha}\right\}=O\left(\exp \left(-c n^{1-(d+1) \alpha / 2}\right)\right) .
$$

Indeed, for a fixed $\alpha \in(0,2 /(d+1))$ we have

$$
\mathrm{P}\left\{S_{n} \leq 1-n^{-\alpha}\right\}=\left(1-\frac{V_{d}\left(\left\{x \in \mathbb{B}^{d}: x \cdot u_{0}>1-n^{-\alpha}\right\}\right)}{\omega_{d}}\right)^{n},
$$

where $V_{d}$ is the Lebesgue measure in $\mathbb{R}^{d}$ and, recall, $\omega_{d}$ is the volume of the unit ball $\mathbb{B}^{d}$. An elementary computation yields

$$
\begin{aligned}
V_{d}\left(\left\{x \in \mathbb{B}^{d}: x \cdot u_{0}>1-n^{-\alpha}\right\}\right) & =\omega_{d-1} \int_{0}^{\arccos \left(1-n^{-\alpha}\right)} \sin ^{d}(\theta) \mathrm{d} \theta \\
& \sim \frac{\omega_{d-1}}{d+1} 2^{(d+1) / 2} n^{-\alpha(d+1) / 2}, \quad n \rightarrow \infty .
\end{aligned}
$$

By combining (6) with (7), we recover (5). 
For a fixed $\alpha \in(0,2 /(d+1))$, we now consider a deterministic covering of the sphere $\mathbb{S}^{d-1}$ by spherical caps of height $n^{-\alpha}$ (i.e. of angular radius $\arccos \left(1-n^{-\alpha}\right)$ ), such that the total number of caps is of order $\Theta\left(n^{\alpha(d-1) / 2}\right)$. In addition, we suppose that every cap intersects at most a fixed number, $\varsigma$, of other caps. Note that the existence of such a covering can be proved by induction on the dimension, $d$.

Indeed, let us suppose that, for every $\varepsilon>0$, there exists a covering of $\mathbb{S}^{d-1}$ by $N_{d}^{\mathrm{cap}}(\varepsilon)$ spherical caps of angular radius $\varepsilon$. We can then construct a covering of the cylinder $\mathcal{C}=$ $\mathbb{S}^{d-1} \times[-\pi / 2, \pi / 2] \subseteq \mathbb{R}^{d+1}$ as follows. For every integer $k$ with $|k| \leq\lfloor\pi / \varepsilon\rfloor$, we use the induction hypothesis to choose $N_{d}^{\text {cap }}(\varepsilon / 2)$ caps, of radius $\varepsilon / 2$ and respectively centred at $a_{i}^{k}$, $1 \leq i \leq N_{d}^{\mathrm{cap}}(\varepsilon / 2)$, on and fully covering the $(d-1)$-dimensional sphere $\mathbb{S}^{d-1} \times\{k \varepsilon / 2\}$. A covering of the set $\mathcal{C}$ by $(2\lfloor\pi / \varepsilon\rfloor+1) N_{d}^{\text {cap }}(\varepsilon / 2)$ balls is then obtained by considering the balls of radius $\varepsilon$ centred at the points $a_{i}^{j}, 1 \leq i \leq N_{d}^{\text {cap }}(\varepsilon / 2),-\lfloor\pi / \varepsilon\rfloor \leq j \leq\lceil\pi / \varepsilon\rceil$. (Here, $\lfloor\cdot\rfloor$ and $\lceil\cdot\rceil$ are the floor and ceiling functions, respectively.) To proceed, note that the mapping $(u, \theta) \mapsto$ $\left(u \sin \theta+e_{d+1} \cos \theta\right)$, where $e_{d+1}=(0, \ldots, 0,1) \in \mathbb{R}^{d+1}$, is a surjection from $\mathcal{C}$ onto the unit sphere $\mathbb{S}^{d} \subseteq \mathbb{R}^{d+1}$ satisfying the Lipschitz condition with constant 1 . This observation allows us to transform the above covering of $\mathcal{C}$ into a covering of $\mathbb{S}^{d}$ by $(2\lfloor\pi / \varepsilon\rfloor+1) N_{d}^{\text {cap }}(\varepsilon / 2)$ caps of radius $\varepsilon$. By induction, it follows that $\mathbb{S}^{d-1}$ can be covered with $\Theta\left(\varepsilon^{d-1}\right)$ spherical caps of radius $\varepsilon$. Moreover, the proof above also shows that the covering thus constructed satisfies the requirement that every cap intersects at most a fixed number, $\varsigma$, of other caps.

Let $\mathscr{D}_{n}$ be the event that the set $\left\{U_{1}, \ldots, U_{n}\right\}$ intersects the interiors of all the caps in the covering. Since the number of caps is polynomial in $n^{\alpha / 2}$, and the probability that $\left\{U_{1}, \ldots, U_{n}\right\}$ does not intersect one cap is bounded subexponentially by the estimate (5), we obtain

$$
\mathrm{P}\left(\mathscr{D}_{n}^{\mathrm{c}}\right)=O\left(\exp \left(-c n^{1-(d+1) \alpha / 2}\right)\right),
$$

where $c$ is a positive constant. In order to prove (4), it remains to note that there exists a positive constant $K$ such that the hyperplanes spanned by the facets of a polyhedron with a vertex in each cap are at least a distance $\left(1-K n^{-\alpha}\right)$ from the origin; thus

$$
\mathscr{D}_{n} \subset\left\{C_{n} \supset B\left(1-K n^{-\alpha}\right)\right\} .
$$

Note that $K$ can be taken to equal 4. Indeed, the interior of any circular cap of height $4 n^{-\alpha}$ contains at least one cap, of height $n^{-\alpha}$, of the initial covering (since the angular radius of the larger cap, $\arccos \left(1-4 n^{-\alpha}\right)$, is greater than the angular diameter of the smaller cap, $2 \arccos \left(1-n^{-\alpha}\right)$ ). Being in $\mathscr{D}_{n}$ then implies that any cap of height $4 n^{-\alpha}$ contains a point of $\left\{U_{1}, \ldots, U_{n}\right\}$ in its interior and, consequently, that any facet of the convex hull of $\left\{U_{1}, \ldots, U_{n}\right\}$ is at least a distance $\left(1-4 n^{-\alpha}\right)$ from the origin. This completes the argument.

Proof of part (ii). If we replace (1) by the equality

$$
\mathrm{P}\left\{\tilde{S}_{t} \leq 1-t^{-\alpha}\right\}=\exp \left(-t V_{d}\left(\left\{x \in \mathbb{B}^{d}: x \cdot u_{0}>1-t^{-\alpha}\right\}\right)\right),
$$

where $\tilde{S}_{t}=\sup _{x \in X_{t}}\left(x \cdot u_{0}\right)$, then the proof of Proposition 1 for the Poisson point process is very similar to that for the binomial point process. We thus omit the details.

\section{Proof of Theorem 1}

An important obstacle in the study of the number of vertices of convex hulls of large samples is that the addition of a new vertex may cause an arbitrarily large number of other vertices to be lost. The idea underlying the proof of Theorem 1 is to circumvent this difficulty by providing 
an appropriate artificial modification of the number-of-vertices functional $N_{n}$ that, while being very similar to $N_{n}$, is better behaved, in that it satisfies a Lipschitz-type condition allowing appropriate concentration-of-measure tools to be applied.

To proceed with this construction, we choose $\alpha \in(0,2 /(d+1))$ and $\beta \in(1-\alpha(d+1) / 2,1)$ and construct a functional $N_{n}^{\alpha, \beta}$ in the following way. By using the same type of covering as in the proof of Proposition 1 , we can cover the shell $\mathbb{B}^{d} \backslash B\left(1-K n^{-\alpha}\right.$ ) (with $K$ as given in Proposition 1) with a number, of order $\Theta\left(n^{\alpha((d+1) / 2-1)}\right)$, of equal-sized spherical caps, each of volume $\Theta\left(n^{-\alpha(d+1) / 2}\right)$ and such that, from each point of $\mathbb{B}^{d} \backslash B\left(1-K n^{-\alpha}\right)$, only a fixed number, $\varsigma$, of caps are seen within $\mathbb{B}^{d} \backslash B\left(1-K n^{-\alpha}\right)$. For a sample, $\mathcal{X}$, in $\mathbb{B}^{d}$, within each of the spherical caps, $\Xi$, constructed above we observe the subsample $\Xi \cap \mathcal{X}$ and, if card $(\Xi \cap \mathcal{X})>n^{\beta}$ (in which case we say that $\Xi$ is overfull), we order the points of $\Xi \cap \mathcal{X}$ in some deterministic way (e.g. by decreasing distance to the origin) and reject those whose rank order exceeds $n^{\beta}$. We shall refer to this procedure as 'overfull-rejection'. We also reject all the sample points falling outside $\mathbb{B}^{d} \backslash B\left(1-K n^{-\alpha}\right)$. Note that a sample point of $\mathcal{X}$ can be rejected more than once if it belongs to several different caps. Writing $\hat{X}$ for the so-reduced sample, we define the functional $\Phi_{n}^{\alpha, \beta}(\mathcal{X})$ to be the number of vertices of the convex hull of $\hat{X}, \operatorname{conv}(\hat{X})$. A crucial observation is that the addition or removal (and, consequently, also the repositioning) of a single sample point of $\mathcal{X}$ can change the value of $\Phi_{n}^{\alpha, \beta}(\mathcal{X})$, by at most $\Theta\left(n^{\beta}\right)$. To see this, note first that when adding a new point, $x$, we encounter the following four possibilities.

- $x \in \operatorname{conv}(\hat{\mathcal{X}})$ and $x$ does not fall into an overfull region; in this case the value of $\Phi_{n}^{\alpha, \beta}$ remains unchanged.

- $x \in \operatorname{conv}(\hat{X})$ but $x$ falls into an overfull region. If $x$ is rejected, $\Phi_{n}^{\alpha, \beta}$ does not change; otherwise, $x$ causes overfull-rejection of another point and might itself become a new vertex of the convex hull of the reduced sample, possibly causing some vertices of $\operatorname{conv}(\hat{X})$ to be lost. Each of these changes results in $\Phi_{n}^{\alpha, \beta}$ changing by at most $\Theta\left(n^{\beta}\right)$, because at most $\Theta\left(n^{\beta}\right)$ points of the reduced sample can be seen from any given point of $\mathbb{B}^{d} \backslash B\left(1-K n^{-\alpha}\right)$.

- $x \notin \operatorname{conv}(\hat{X})$ and $x$ does not fall into an overfull region; in this case $x$ becomes a new vertex, causing at most $\Theta\left(n^{\beta}\right)$ vertices of $\hat{X}$ to be lost.

- $x \notin \operatorname{conv}(\hat{X})$ and $x$ does fall into an overfull region. If $x$ itself is rejected, nothing changes; otherwise, $x$ becomes a new vertex, possibly causing overfull-rejection of another vertex of $\operatorname{conv}(\hat{X})$ and possibly causing some vertices of $\operatorname{conv}(\hat{X})$ to be lost. As above, each of these changes results in $\Phi_{n}^{\alpha, \beta}$ changing by at most $\Theta\left(n^{\beta}\right)$, since at most $\Theta\left(n^{\beta}\right)$ points of the reduced sample can be seen from any given point of $\mathbb{B}^{d} \backslash B\left(1-K n^{-\alpha}\right)$.

A similar argument shows that the removal of a sample point also results in an overall change of $\Phi_{n}^{\alpha, \beta}$, by at most $\Theta\left(n^{\beta}\right)$.

To define $N_{n}^{\alpha, \beta}$, we henceforth let

$$
N_{n}^{\alpha, \beta}:=\Phi_{n}^{\alpha, \beta}\left(\left\{U_{1}, \ldots, U_{n}\right\}\right) .
$$

The proof of Theorem 1 is divided into three steps. Using Proposition 1, in Lemma 1 we show that $N_{n}^{\alpha, \beta}$ is a good approximation of $N_{n}$. In Lemma 2 we then give some concentration properties of the number of points of the sample $\left\{U_{1}, \ldots, U_{n}\right\}$ that fall into the annulus $\mathbb{B}^{d} \backslash B\left(1-K n^{-\alpha}\right)$. In Lemma 3, which is the key result of our proof, we deduce from 
Lemma 2 and a classical concentration-of-measure result a large deviation property of $N_{n}^{\alpha, \beta}$. Theorem 1 is then easily concluded from Lemmas 1 and 3.

Lemma 1. There exists a positive constant, $c$, such that

$$
\mathrm{P}\left\{N_{n} \neq N_{n}^{\alpha, \beta}\right\} \leq O\left(\exp \left(-c n^{1-\alpha(d+1) / 2}\right)\right) .
$$

In particular,

$$
\left|\mathrm{E}\left(N_{n}^{\alpha, \beta}\right)-\mathrm{E}\left(N_{n}\right)\right| \leq n \mathrm{P}\left\{N_{n} \neq N_{n}^{\alpha, \beta}\right\}=O\left(\exp \left(-c n^{1-\alpha(d+1) / 2}\right)\right) .
$$

Proof. Let $\mathcal{A}_{n}$ and $\mathscr{B}_{n}$ be the events that, respectively, there is at least one extreme point of $\left\{U_{1}, \ldots, U_{n}\right\}$ in $B\left(1-K n^{-\alpha}\right)$ and there is at least one spherical cap containing more than $n^{\beta}$ points.

Using Proposition 1, we have

$$
\mathrm{P}\left(\mathscr{A}_{n}\right)=O\left(\exp \left(-c n^{1-\alpha(d+1) / 2}\right)\right) .
$$

Moreover, denoting by $\operatorname{bin}(n, p)$ a binomial variable with parameters $n$ and $p$, we obtain

$$
\mathrm{P}\left(\mathcal{B}_{n}\right) \leq \Psi_{n} \mathrm{P}\left\{\operatorname{bin}\left(n, v_{n}\right) \geq n^{\beta}\right\},
$$

where $\Psi_{n}$ is the number of spherical caps and $v_{n}$ is the Lebesgue measure of a single cap divided by $\omega_{d}$. Using the Legendre transform, we have

$$
\begin{aligned}
\mathrm{P}\left\{\operatorname{bin}\left(n, v_{n}\right) \geq n^{\beta}\right\} \leq & \inf _{t \geq 0}\left\{\mathrm{e}^{-t n^{\beta}} \mathrm{E}\left(\mathrm{e}^{t \operatorname{bin}\left(n, v_{n}\right)}\right)\right\} \\
= & \inf _{t \geq 0}\left\{\exp \left(-t n^{\beta}+n \log \left(\mathrm{e}^{t} v_{n}+1-v_{n}\right)\right)\right\} \\
= & \exp \left(n \log \left(\left(1-v_{n}\right) \frac{n^{\beta}}{n-n^{\beta}}+1-v_{n}\right)\right. \\
& \left.\quad-n^{\beta} \log \left(\left(v_{n}^{-1}-1\right) \frac{n^{\beta}}{n-n^{\beta}}\right)\right),
\end{aligned}
$$

where the last equality is obtained by setting $t=\log \left(\left(v_{n}^{-1}-1\right) n^{\beta} /\left(n-n^{\beta}\right)\right)$.

Since $v_{n}=\Theta\left(n^{-\alpha(d+1) / 2}\right)$, it follows that

$$
\mathrm{P}\left\{\operatorname{bin}\left(n, v_{n}\right) \geq n^{\beta}\right\}=O\left(\mathrm{e}^{-c n^{\beta}}\right) .
$$

By combining (9) with the estimate $\Psi_{n}=O\left(n^{\alpha(d-1) / 2}\right)$, we deduce that

$$
\mathrm{P}\left(\mathcal{B}_{n}\right)=O\left(\mathrm{e}^{-c n^{\beta}}\right) .
$$

Furthermore, from the definition of $N_{n}^{\alpha, \beta}$ it follows that

$$
\mathrm{P}\left\{N_{n} \neq N_{n}^{\alpha, \beta}\right\} \leq \mathrm{P}\left(\mathcal{A}_{n}\right)+\mathrm{P}\left(\mathscr{B}_{n}\right) .
$$

Inserting the estimations (8) and (10) into (11) completes the proof of Lemma 1.

Let $M_{n}$ be the number of $U_{i}, 1 \leq i \leq n$, contained in $\mathbb{B}^{d} \backslash B\left(1-K n^{-\alpha}\right) ; M_{n}$ then has a binomial distribution with parameters $n$ and $w_{n}$, where $w_{n}=V_{d}\left(\mathbb{B}^{d} \backslash B\left(1-K n^{-\alpha}\right)\right) / \omega_{d}$. In the following lemma we collect some technical estimates for $M_{n}$ needed for the proof of Lemma 3. 
Lemma 2. With $k_{n}^{-}=\left\lfloor n w_{n}-n^{1-2 /(d+1)}\right\rfloor$ and $k_{n}^{+}=\left\lceil n w_{n}+n^{1-2 /(d+1)}\right\rceil$, we have

$$
\begin{gathered}
\liminf _{n \rightarrow \infty} \frac{1}{\log (n)} \log \left(-\log \mathrm{P}\left\{M_{n} \notin\left[k_{n}^{-}, k_{n}^{+}\right]\right\}\right) \geq 1+\alpha-\frac{4}{d+1}, \\
\lim _{n \rightarrow \infty} \sup _{k \in\left[k_{n}^{-}, k_{n}^{+}\right]}\left|\frac{\mathrm{E}\left(N_{n}^{\alpha, \beta} \mid M_{n}=k\right)}{\mathrm{E}\left(N_{n}^{\alpha, \beta}\right)}-1\right|=0 .
\end{gathered}
$$

Proof. To prove (12) we use Chebyshev's inequality to obtain

$$
\begin{aligned}
\mathrm{P}\left\{M_{n} \notin\left[k_{n}^{-}, k_{n}^{+}\right]\right\} \leq & \inf _{u \geq 1}\left\{u^{-k_{n}^{+}}\left(w_{n} u+1-w_{n}\right)^{n}\right\}+\inf _{0<v<1}\left\{v^{-k_{n}^{-}}\left(w_{n} v+1-w_{n}\right)^{n}\right\} \\
\leq & \left(1+\frac{n^{-2 /(d+1)}}{w_{n}}\right)^{-k_{n}^{+}}\left(1+n^{-2 /(d+1)}\right)^{n} \\
& +\left(1-\frac{n^{-2 /(d+1)}}{w_{n}}\right)^{-k_{n}^{-}}\left(1-n^{-2 /(d+1)}\right)^{n},
\end{aligned}
$$

where $u:=1+n^{-2 /(d+1)} / w_{n}$ and $v:=1-n^{-2 /(d+1)} / w_{n}$. Taking logarithms of both sides yields

$$
\begin{aligned}
&-\log \mathrm{P}\left\{M_{n} \notin\left[k_{n}^{-}, k_{n}^{+}\right]\right\} \\
& \geq-\log 2-\max \left\{n \log \left(1+n^{-2 /(d+1)}\right)-k_{n}^{+} \log \left(1+\frac{n^{-2 /(d+1)}}{w_{n}}\right),\right. \\
&\left.n \log \left(1-n^{-2 /(d+1)}\right)-k_{n}^{-} \log \left(1-\frac{n^{-2 /(d+1)}}{w_{n}}\right)\right\} .
\end{aligned}
$$

Thus, applying the second-order Taylor expansion $\log (1+x)=x-x^{2} / 2+o\left(x^{2}\right)$ yields

$$
\begin{aligned}
& -\log \mathrm{P}\left\{M_{n} \notin\left[k_{n}^{-}, k_{n}^{+}\right]\right\} \\
& \geq-\log 2 \\
& \quad-\max \left\{n\left(n^{-2 /(d+1)}+O\left(n^{-4 /(d+1)}\right)\right)\right. \\
& \quad-\left[n w_{n}+n^{1-2 /(d+1)}\right]\left(\frac{n^{-2 /(d+1)}}{w_{n}}-\frac{n^{-4 /(d+1)}}{2 w_{n}^{2}}+o\left(\frac{n^{-4 /(d+1)}}{w_{n}^{2}}\right)\right), \\
& n\left(-n^{-2 /(d+1)}+O\left(n^{-4 /(d+1)}\right)\right) \\
& \left.\quad-\left[n w_{n}-n^{1-2 /(d+1)}\right]\left(-\frac{n^{-2 /(d+1)}}{w_{n}}-\frac{n^{-4 /(d+1)}}{2 w_{n}^{2}}+o\left(\frac{n^{-4 /(d+1)}}{w_{n}^{2}}\right)\right)\right\} \\
& =\frac{n^{1-4 /(d+1)}}{2 w_{n}}[1+o(1)]+O\left(n^{1-4 /(d+1)}\right) .
\end{aligned}
$$

By using the estimate $w_{n} \sim d K n^{-\alpha}$ as $n \rightarrow \infty$, we obtain the asymptotic result (12), as required.

To prove (13), for $k \in \mathbb{N}$ respectively denote by $\hat{C}_{k}^{n}$ and $N_{n, k}$ the convex hull and the number of extreme points of $k$ points independently and identically uniformly distributed in 
$\mathbb{B}^{d} \backslash B\left(1-K n^{-\alpha}\right)$. Conditional on $\left\{M_{n}=k\right\}$, when the convex hull $\hat{C}_{k}^{n}$ is strictly smaller than $C_{n}$ the latter does not contain $\mathbb{B}^{d} \backslash B\left(1-K n^{-\alpha}\right)$. Consequently, we have

$$
0 \leq \mathrm{E}\left(N_{n} \mid M_{n}=k\right)-\mathrm{E}\left(N_{n, k}\right) \leq \mathrm{E}\left(N_{n} \mathbf{1}_{\mathcal{A}_{n}} \mid M_{n}=k\right) \leq n \mathrm{P}\left(\mathcal{A}_{n} \mid M_{n}=k\right),
$$

where $\mathcal{A}_{n}=\left\{C_{n} \not \supset B\left(1-K n^{-\alpha}\right)\right\}$ is the event defined at the beginning of the proof of Lemma 1.

As in the proof of Proposition 1(i) (i.e. $\lim _{n \rightarrow \infty} \mathrm{P}\left\{\mathcal{A}_{n}\right\}=0$ ), the covering of

$$
\mathbb{B}^{d} \backslash B\left(1-K n^{-\alpha}\right)
$$

with $\Psi_{n}$ spherical caps can be used to deduce that

$$
\begin{array}{r}
\mathrm{P}\left(\mathcal{A}_{n} \mid M_{n}=k\right) \leq \Psi_{n}\left(1-\frac{V_{d}\left(\left\{x \in \mathbb{B}^{d} \backslash B\left(1-K n^{-\alpha}\right): x \cdot u_{0}>1-n^{-\alpha}\right\}\right)}{V_{d}\left(\mathbb{B}^{d} \backslash B\left(1-K n^{-\alpha}\right)\right)}\right)^{k_{n}^{-}}, \\
u_{0} \in \mathbb{S}^{d-1} .
\end{array}
$$

Following the proof of Proposition 1(i) further, we combine this inequality with (14) to obtain

$$
\lim _{n \rightarrow \infty} \sup _{k \in\left[k_{n}^{-}, k_{n}^{+}\right]}\left\{\mathrm{E}\left(N_{n} \mid M_{n}=k\right)-\mathrm{E}\left(N_{n, k}\right)\right\}=\lim _{n \rightarrow \infty} n \sup _{k \in\left[k_{n}^{-}, k_{n}^{+}\right]} \mathrm{P}\left(\mathcal{A}_{n} \mid M_{n}=k\right)=0 .
$$

Using Efron's equality (Equation (3.7) of [6]) for $C_{n-1}$ and $\hat{C}_{k-1}^{n}$, we then obtain

$$
\frac{\mathrm{E}\left(N_{n}\right)}{\mathrm{E}\left(N_{n, k}\right)}=\frac{n}{k}\left(1-\frac{\mathrm{E}\left(V_{d}\left(C_{n-1}\right)\right)}{\omega_{d}}\right)\left(1-\frac{\mathrm{E}\left(V_{d}\left(\hat{C}_{k-1}^{n} \backslash B\left(1-K n^{-\alpha}\right)\right)\right)}{V_{d}\left(\mathbb{B}^{d} \backslash B\left(1-K n^{-\alpha}\right)\right)}\right)^{-1} .
$$

Upon combining (16) with (15), it follows that, uniformly in $k \in\left[k_{n}^{-}, k_{n}^{+}\right]$,

$$
\frac{\mathrm{E}\left(N_{n}\right)}{\mathrm{E}\left(N_{n} \mid M_{n}=k\right)}=\frac{n}{k}\left(1-\frac{\mathrm{E}\left(V_{d}\left(C_{n-1}\right)\right)}{\omega_{d}}\right)\left(1-\frac{\mathrm{E}\left(V_{d}\left(\hat{C}_{k-1}^{n} \backslash B\left(1-K n^{-\alpha}\right)\right)\right)}{V_{d}\left(\mathbb{B}^{d} \backslash B\left(1-K n^{-\alpha}\right)\right)}\right)^{-1}+o(1) .
$$

We claim that

$$
\mathrm{E}\left(V_{d}\left(\mathbb{B}^{d} \backslash\left[\hat{C}_{k-1}^{n} \cup B\left(1-K n^{-\alpha}\right)\right]\right)\right) \sim \mathrm{E}\left(V_{d}\left(\mathbb{B}^{d} \backslash C_{n-1}\right)\right),
$$

uniformly for $k \in\left[k_{n}^{-}, k_{n}^{+}\right]$. Indeed, by using Proposition 1 and (12) we see that

$\mathrm{E}\left(V_{d}\left(\mathbb{B}^{d} \backslash C_{n-1}\right)\right)=\mathrm{E}\left(\mathrm{E}\left(V_{d}\left(\mathbb{B}^{d} \backslash\left[\hat{C}_{M_{n}-1}^{n} \cup B\left(1-K n^{-\alpha}\right)\right]\right) \mid M_{n}\right) \mathbf{1}_{\left\{M_{n} \in\left[k_{n}^{-}, k_{n}^{+}\right]\right\}}\right)(1+o(1))$.

Then, taking into account the fact that $\mathrm{E}\left(V_{d}\left(\mathbb{B}^{d} \backslash\left[\hat{C}_{k-1}^{n} \cup B\left(1-K n^{-\alpha}\right)\right]\right)\right)$ decreases with $k$, we conclude that

$$
\begin{aligned}
\mathrm{E}\left(V_{d}\right. & \left.\left(\mathbb{B}^{d} \backslash\left[\hat{C}_{k_{n}^{+}-1}^{n} \cup B\left(1-K n^{-\alpha}\right)\right]\right)\right)(1+o(1)) \\
& \leq \mathrm{E}\left(V_{d}\left(\mathbb{B}^{d} \backslash C_{n-1}\right)\right) \\
& \leq \mathrm{E}\left(V_{d}\left(\mathbb{B}^{d} \backslash\left[\hat{C}_{k_{n}^{-}-1}^{n} \cup B\left(1-K n^{-\alpha}\right)\right]\right)\right)(1+o(1)) .
\end{aligned}
$$

However, $\mathrm{E}\left(V_{d}\left(\mathbb{B}^{d} \backslash\left[\hat{C}_{k-1}^{n} \cup B\left(1-K n^{-\alpha}\right)\right]\right)\right)$ decreases with $n$. For $n^{\prime}>n$ this can be seen by coupling the $k-1$ independent, identically distributed points $U_{1}, \ldots, U_{k-1}$, which are 
uniform in $\mathbb{B}^{d} \backslash B\left(1-K n^{-\alpha}\right)$, to the points $U_{1}^{\prime}, \ldots, U_{k-1}^{\prime}$ given by $U_{i}^{\prime}:=\vartheta\left(\left|U_{i}\right|\right) U_{i} /\left|U_{i}\right|$. Here $\vartheta(\cdot) \equiv \vartheta_{n, n^{\prime} ; \alpha}(\cdot)$ is a function of the form $\vartheta(r)=c_{1}\left(r^{d}+c_{2}\right)^{1 / d}$, with $c_{1}$ and $c_{2}$ chosen such that $\vartheta(1)=1$ and $\vartheta\left(1-K n^{-\alpha}\right)=1-K n^{\prime-\alpha}$. The random points $U_{i}^{\prime}$ can readily be verified to be independent and identically uniformly distributed in $\mathbb{B}^{d} \backslash B\left(1-K n^{\prime-\alpha}\right)$ and to have the property that, for each $i, U_{i}^{\prime}$ is almost surely closer to the boundary $\partial \mathbb{B}^{d}$ than is $U_{i}$. By combining these observations with (19), and choosing $m_{n}^{+}$and $m_{n}^{-}, m_{n}^{+}>m_{n}^{-}$, such that $k_{n}^{+}=k_{m_{n}^{+}}^{-}$and $k_{n}^{-}=k_{m_{n}^{-}}^{+}$, we see that

$$
\begin{aligned}
\mathrm{E}\left(V_{d}\left(\mathbb{B}^{d} \backslash C_{m_{n}^{+}-1}\right)\right)(1+o(1)) & \leq \mathrm{E}\left(V_{d}\left(\mathbb{B}^{d} \backslash\left[\hat{C}_{k-1}^{n} \cup B\left(1-K n^{-\alpha}\right)\right]\right)\right) \\
& \leq \mathrm{E}\left(V_{d}\left(\mathbb{B}^{d} \backslash C_{m_{n}^{-}-1}\right)\right)(1+o(1)),
\end{aligned}
$$

uniformly for $k \in\left[k_{n}^{-}, k_{n}^{+}\right]$. Since $m_{n}^{+} \sim m_{n}^{-}$, this yields (18) as an immediate consequence. We rewrite (18) as

$$
V_{d}\left(\mathbb{B}^{d} \backslash B\left(1-K n^{-\alpha}\right)\right)-\mathrm{E}\left(V_{d}\left(\hat{C}_{k-1}^{n} \backslash B\left(1-K n^{-\alpha}\right)\right)\right) \sim \omega_{d}-\mathrm{E}\left(V_{d}\left(C_{n-1}\right)\right),
$$

uniformly in $k \in\left[k_{n}^{-}, k_{n}^{+}\right]$. Combining this relation with (17) yields

$$
\lim _{n \rightarrow \infty} \sup _{k \in\left[k_{n}^{-}, k_{n}^{+}\right]}\left|\frac{\mathrm{E}\left(N_{n} \mid M_{n}=k\right)}{\mathrm{E}\left(N_{n}\right)}-1\right|=0 .
$$

In order to deduce (13), it only remains to apply the method of Lemma 1, to obtain

$$
\begin{aligned}
\sup _{k_{n}^{-} \leq k \leq k_{n}^{+}}\left|\mathrm{E}\left(N_{n}^{\alpha, \beta} \mid M_{n}=k\right)-\mathrm{E}\left(N_{n} \mid M_{n}=k\right)\right| & \leq 2 n \sup _{k_{n}^{-} \leq k \leq k_{n}^{+}} \mathrm{P}\left(\mathcal{A}_{n} \cup \mathcal{B}_{n} \mid M_{n}=k\right) \\
& =O\left(\exp \left(-c n^{1-\alpha(d+1) / 2}\right)\right) .
\end{aligned}
$$

This completes the proof of Lemma 2.

The next lemma, which is an essential step in obtaining Theorem 1, shows how the Lipschitz property of the function $\Phi_{n}^{\alpha, \beta}$ can be used to estimate large deviation probabilities for $N_{n}^{\alpha, \beta}$.

Lemma 3. For each $\varepsilon>0$,

$$
\liminf _{n \rightarrow \infty} \frac{1}{\log (n)} \log \left(-\log \mathrm{P}\left\{\left|\frac{N_{n}^{\alpha, \beta}}{\mathrm{E}\left(N_{n}^{\alpha, \beta}\right)}-1\right|>\varepsilon\right\}\right) \geq 1+\alpha-\frac{4}{d+1}-2 \beta .
$$

Proof. Conditional on $\left\{M_{n}=k\right\}, 0 \leq k \leq n$, the variable $N_{n}^{\alpha, \beta}$ is distributed in the same way as $\Phi_{n}^{\alpha, \beta}\left(Y_{1}, \ldots, Y_{k}\right)$, where $Y_{1}, \ldots, Y_{k}$ are independent, uniformly distributed variables in $\mathbb{B}^{d} \backslash B\left(1-K n^{-\alpha}\right)$.

Taking into account our discussion of the properties of $\Phi_{n}^{\alpha, \beta}$ at the beginning of this section,

$$
\Phi_{n}^{\alpha, \beta}:\left(\mathbb{B}^{d} \backslash B\left(1-K n^{-\alpha}\right)\right)^{k} \rightarrow \mathbb{N}
$$

is a Lipschitz function with constant $c n^{\beta}, c>0$, for $\left(\mathbb{B}^{d} \backslash B\left(1-K n^{-\alpha}\right)\right)^{k}$ endowed with the metric

$$
\rho_{k}\left(\left(x_{1}, \ldots, x_{k}\right),\left(x_{1}^{\prime}, \ldots, x_{k}^{\prime}\right)\right):=\mathbf{1}_{\left\{x_{1} \neq x_{1}^{\prime}\right\}}+\cdots+\mathbf{1}_{\left\{x_{k} \neq x_{k}^{\prime}\right\}} .
$$

Consequently, we are in a position to apply the following standard concentration-of-measure result. 
Theorem 3. ([8, Corollary 1.17].) Let $Y_{1}, Y_{2}, \ldots, Y_{k}$ be independent random variables taking values in a metric space, $(\mathcal{y}, \rho)$, of finite diameter $D$. Assume that $\Phi: y^{k} \rightarrow \mathbb{R}$ is Lipschitz with respect to the $L^{1}$-metric $\rho_{k}\left(\left(y_{1}, \ldots, y_{k}\right),\left(y_{1}^{\prime}, \ldots, y_{k}^{\prime}\right)\right):=\rho\left(y_{1}, y_{1}^{\prime}\right)+\cdots+\rho\left(y_{k}, y_{k}^{\prime}\right)$, with some Lipschitz constant $L$. Then, for every $\lambda \geq 0$,

$$
\mathrm{P}\left\{\left|\Phi\left(Y_{1}, \ldots, Y_{k}\right)-\mathrm{E}\left(\Phi\left(Y_{1}, \ldots, Y_{k}\right)\right)\right|>\lambda\right\} \leq 2 \exp \left(-\frac{\lambda^{2}}{2 k L^{2} D^{2}}\right) .
$$

By applying Theorem 3 to $\lambda=\varepsilon \mathrm{E}\left(N_{n}^{\alpha, \beta} \mid M_{n}=k\right)$, for every $k, 0 \leq k \leq n$, we obtain

$$
\begin{aligned}
& \mathrm{P}\left\{\left|\frac{N_{n}^{\alpha, \beta}}{\mathrm{E}\left(N_{n}^{\alpha, \beta}\right)}-1\right|>\varepsilon \mid M_{n}=k\right\} \\
& \quad \leq \mathrm{P}\left\{\left|N_{n}^{\alpha, \beta}-\mathrm{E}\left(N_{n}^{\alpha, \beta} \mid M_{n}=k\right)\right|>\varepsilon \mathrm{E}\left(N_{n}^{\alpha, \beta}\right)-\left|\mathrm{E}\left(N_{n}^{\alpha, \beta} \mid M_{n}=k\right)-\mathrm{E}\left(N_{n}^{\alpha, \beta}\right)\right|\right. \\
& \left.\quad \leq 2 \exp \left(-\frac{\left[\varepsilon \mathrm{E}\left(N_{n}^{\alpha, \beta}\right)-\mid \mathrm{E}\left(N_{n}^{\alpha, \beta} \mid M_{n}=k\right\}\right.}{2 c^{2} k n^{2 \beta}}\right) . \mathrm{E}\left(N_{n}^{\alpha, \beta}\right) \mid\right]^{2} \\
&
\end{aligned}
$$

By applying (13), we deduce from (20) that there exists a positive constant $c$ such that

$$
\sup _{k_{n}^{-} \leq k \leq k_{n}^{+}} \mathrm{P}\left\{\left|\frac{N_{n}^{\alpha, \beta}}{\mathrm{E}\left(N_{n}^{\alpha, \beta}\right)}-1\right|>\varepsilon \mid M_{n}=k\right\} \leq 2 \exp \left(-\frac{c \mathrm{E}\left(N_{n}^{\alpha, \beta}\right)^{2}}{n^{1+2 \beta} w_{n}}\right) .
$$

By now combining (21) with Lemma 1 , the estimate $w_{n} \sim c n^{-\alpha}$, and the classical result $\mathrm{E}\left(N_{n}\right) \sim c n^{1-2 /(d+1)}$ (see [11]), we obtain

$$
\begin{aligned}
& \liminf _{n \rightarrow \infty} \frac{1}{\log (n)} \log \left(-\log \left(\sup _{k_{n}^{-} \leq k \leq k_{n}^{+}} \mathrm{P}\left\{\left|\frac{N_{n}^{\alpha, \beta}}{\mathrm{E}\left(N_{n}^{\alpha, \beta}\right)}-1\right|>\varepsilon \mid M_{n}=k\right\}\right)\right) \\
& \quad \geq \liminf _{n \rightarrow \infty} \frac{1}{\log (n)} \log \left(-\log \left(\exp \left(-c \frac{\mathrm{E}\left(N_{n}\right)^{2}}{n^{1+2 \beta-\alpha}}\right)\right)\right) \\
& \quad=1+\alpha-\frac{4}{d+1}-2 \beta .
\end{aligned}
$$

Furthermore, note that

$$
\begin{aligned}
\mathrm{P}\left\{\left|\frac{N_{n}^{\alpha, \beta}}{\mathrm{E}\left(N_{n}^{\alpha, \beta}\right)}-1\right|>\varepsilon\right\} \leq & \sum_{\substack{k_{n}^{-} \leq k \leq k_{n}^{+}\\
}} \mathrm{P}\left\{\left|\frac{N_{n}^{\alpha, \beta}}{\mathrm{E}\left(N_{n}^{\alpha, \beta}\right)}-1\right|>\varepsilon \mid M_{n}=k\right\} \mathrm{P}\left\{M_{n}=k\right\} \\
& +\mathrm{P}\left\{M_{n} \notin\left[k_{n}^{-}, k_{n}^{+}\right]\right\} .
\end{aligned}
$$

Inserting (12) and (22) into (23) completes the proof of Lemma 3.

We now complete the proof of Theorem 1 . We have

$$
\mathrm{P}\left\{\left|\frac{N_{n}}{\mathrm{E}\left(N_{n}\right)}-1\right|>\varepsilon\right\} \leq \mathrm{P}\left\{\left|\frac{N_{n}^{\alpha, \beta}}{\mathrm{E}\left(N_{n}^{\alpha, \beta}\right)}-1\right|>\varepsilon \frac{\mathrm{E}\left(N_{n}\right)}{\mathrm{E}\left(N_{n}^{\alpha, \beta}\right)}-\left|\frac{\mathrm{E}\left(N_{n}\right)}{\mathrm{E}\left(N_{n}^{\alpha, \beta}\right)}-1\right|\right\}+\mathrm{P}\left\{N_{n} \neq N_{n}^{\alpha, \beta}\right\} .
$$


From Lemmas 1 and 3 we find that, for every $\alpha \in(0,2 /(d+1))$ and $\beta \in(1-\alpha(d+1) / 2,1)$,

$$
\begin{gathered}
\liminf _{n \rightarrow \infty} \frac{1}{\log (n)} \log \left(-\log \left(\sup _{k_{n}^{-} \leq k \leq k_{n}^{+}} \mathrm{P}\left\{\left|\frac{N_{n}}{\mathrm{E}\left(N_{n}\right)}-1\right|>\varepsilon\right\}\right)\right) \\
\geq \min \left(1-\alpha \frac{d+1}{2}, 1+\alpha-\frac{4}{d+1}-2 \beta\right) .
\end{gathered}
$$

It then remains to verify that

$$
\sup _{\substack{\alpha \in(0,2 /(d+1)) \\ \beta \in(1-\alpha(d+1) / 2,1)}} \min \left(1-\alpha \frac{d+1}{2}, 1+\alpha-\frac{4}{d+1}-2 \beta\right)=\frac{d-1}{3 d+5},
$$

the details of which we omit.

\section{Proof of Theorem 2}

Here the method of proof is similar to that in the binomial case. For some $\alpha \in(0,2 /(d+1))$ and $\beta \in(1-\alpha(d+1) / 2,1)$, we consider a covering of the annulus $\mathbb{B}^{d} \backslash B\left(1-K n^{-\alpha}\right)$ by spherical caps (provided by part (ii) of Proposition 1). In full analogy with the definition of $N_{n}^{\alpha, \beta}$, we define a modification, $\tilde{N}_{t}^{\alpha, \beta}$, of $\tilde{N}_{t}$ by letting $\tilde{N}_{t}^{\alpha, \beta}:=\Phi_{t}^{\alpha, \beta}\left(X_{t}\right)$; thus, $\tilde{N}_{t}^{\alpha, \beta}$ is the number of vertices of the convex hull of an appropriate subset of the intersection of the Poisson point process $X_{t}$ with $\mathbb{B}^{d} \backslash B\left(1-K t^{-\alpha}\right)$. This convex hull has the property that each cap contains at most $t^{\beta}$ points. Much along the same lines as in the proof of Lemma 1, we obtain

$$
\mathrm{P}\left\{\tilde{N}_{t}^{\alpha, \beta} \neq \tilde{N}_{t}\right\}=O\left(\exp \left(-c t^{1-\alpha(d+1) / 2}\right)\right)
$$

and

$$
\left|\mathrm{E}\left(\tilde{N}_{t}^{\alpha, \beta}\right)-\mathrm{E}\left(\tilde{N}_{t}\right)\right|=O\left(\exp \left(-c t^{1-\alpha(d+1) / 2}\right)\right) .
$$

Moreover, let $\tilde{M}_{t}$ be the number of points of $X_{t} \cap\left[B\left(1-K t^{-\alpha}\right)\right]^{\mathrm{c}} ; \tilde{M}_{t}$ is then Poisson-distributed with mean

$$
\mathrm{E}\left(\tilde{M}_{t}\right)=t V_{d}\left(\mathbb{B}^{d} \backslash B\left(1-K t^{-\alpha}\right)\right) \sim d K \omega_{d} t^{1-\alpha}, \quad t \rightarrow \infty .
$$

As for $M_{n}$ in the proof of Lemma 3, $\tilde{M}_{t}$ satisfies a large deviation inequality. In fact, with $k_{t}^{-}=\left\lfloor\mathrm{E}\left(\tilde{M}_{t}\right)-t^{1-2 /(d+1)}\right\rfloor$ and $k_{t}^{+}=\left\lceil\mathrm{E}\left(\tilde{M}_{t}\right)+t^{1-2 /(d+1)}\right\rceil$, as in (12) we obtain

$$
\liminf _{t \rightarrow \infty} \frac{1}{\log (t)} \log \left(-\log \mathrm{P}\left\{\tilde{M}_{t} \notin\left[k_{t}^{-}, k_{t}^{+}\right]\right\}\right) \geq 1+\alpha-\frac{4}{d+1} .
$$

Furthermore, we have

$$
\begin{aligned}
\mathrm{P}\left\{\left|\frac{\tilde{N}_{t}^{\alpha, \beta}}{\mathrm{E}\left(\tilde{N}_{t}^{\alpha, \beta}\right)}-1\right|>\varepsilon\right\} \leq & \sum_{\substack{k_{t}^{-} \leq k \leq k_{t}^{+}\\
}} \mathrm{P}\left\{\left|\frac{\tilde{N}_{t}^{\alpha, \beta}}{\mathrm{E}\left(\tilde{N}_{t}^{\alpha, \beta}\right)}-1\right|>\varepsilon \mid \tilde{M}_{t}=k\right\} \mathrm{P}\left\{\tilde{M}_{t}=k\right\} \\
& +\mathrm{P}\left\{\tilde{M}_{t} \notin\left[k_{t}^{-}, k_{t}^{+}\right]\right\} .
\end{aligned}
$$

The first term can be bounded in the same way as the corresponding one in (23) and the second term can be estimated using (26). Consequently, the large deviation result is proved for $\tilde{N}_{t}^{\alpha, \beta}$, and it suffices to use (24) and (25) to do the same for $\tilde{N}_{t}$. 


\section{Concluding remarks}

Remark 1. Our results remain valid if we add to the random sample in the ball a fixed number of deterministic points. Indeed, the whole argument above can be repeated for such a case with only minor changes.

Remark 2. Since the asymptotic behaviour of the convex hull only depends on the geometry of the sample very close to the boundary of $\mathbb{B}^{d}$, the results of Proposition 1 and Theorem 2 can be extended to the class of inhomogeneous Poisson point processes $Y_{t}$ with intensity measures of the form $t \mathrm{~d} \mu$, where (in spherical coordinates) $\mu$ is the measure $f(r) \mathbf{1}_{[0,1]}(r) \mathrm{d} r \mathrm{~d} \sigma_{d}(u), d \sigma_{d}$ being the area measure on the sphere $\mathbb{S}^{d-1}$ and $f$ a continuous function satisfying $f(1)=1$. Indeed, in a vicinity of $\mathbb{S}^{d-1}$, the intensity measure is close to a multiple of the Lebesgue measure and our argument can again be repeated for this case.

Remark 3. The question of the extension of our results to a general convex set is still open. The method does not apply when the ball is replaced by a polyhedron, since the mean number of extreme points becomes of order $\log (n)$, which is too small for the rates obtained by concentrationof-measure techniques to absorb the polynomial prefactors arising due to deterministic surface partitions, as considered in our proofs.

\section{Acknowledgements}

The second author gratefully acknowledges the support of the Foundation for Polish Science (FNP) and the Polish Ministry of Scientific Research and Information Technology grant 1 P03A 01828 (2005-2007).

\section{References}

[1] Bárány, I. (1992). Random polytopes in smooth convex bodies. Mathematika 39, 81-92.

[2] Bárány, I. and Larman, D. G. (1988). Convex bodies, economic cap coverings, random polytopes. Mathematika 35, 274-291.

[3] Buchta, C. AND Müller, J. (1984). Random polytopes in a ball. J. Appl. Prob. 21, 753-762.

[4] Calka, P. (2002). The distributions of the smallest disks containing the Poisson-Voronoi typical cell and the Crofton cell in the plane. Adv. Appl. Prob. 34, 702-717.

[5] Calka, P. and Schreiber, T. (2005). Limit theorems for the typical Poisson-Voronoi cell and the Crofton cell with a large inradius. Ann. Prob. 33, 1625-1642.

[6] Efron, B. (1965). The convex hull of a random set of points. Biometrika 52, 331-343.

[7] Groeneboom, P. (1988). Limit theorems for convex hulls. Prob. Theory Relat. Fields 79, 327-368.

[8] Ledoux, M. (2001). The Concentration of Measure Phenomenon (Math. Surveys Monogr. 89). American Mathematical Society, Providence, RI.

[9] Massé, B. (2000). On the LLN for the number of vertices of a random convex hull. Adv. Appl. Prob. 32, 675-681.

[10] Reitzner, M. (2003). Random polytopes and the Efron-Stein jackknife inequality. Ann. Prob. 31, $2136-2166$.

[11] RÉNYi, A. AND Sulanke, R. (1963). Über die konvexe Hülle von $n$ zufällig gewählten Punkten. Z. Wahrscheinlichkeitsth. 2, 75-84.

[12] Schreiber, T. (2003). A note on large deviation probabilities for volumes of unions of random closed sets. Submitted. Available at http://www.mat.uni.torun.pl/preprints.

[13] Schütт, C. (1994). Random polytopes and affine surface area. Math. Nachr. 170, 227-249.

[14] Wieacker, J. A. (1978). Einige Probleme der polyedrischen Approximation. Doctoral Thesis, Universität Freiburg. 